\title{
Behavioral Effects of Developmental Exposure to JWH-018 in Wild-Type and Disrupted in Schizophrenia 1 (disc1) Mutant Zebrafish
}

\author{
Judit García-González ${ }^{1}$, Bruno de Quadros ${ }^{1}$, William Havelange ${ }^{1}$, Alistair J. Brock ${ }^{2}$ and Caroline H. Brennan ${ }^{1, * \mathbb{D}}$ \\ 1 School of Biological and Chemical Sciences, Queen Mary, University of London, London E1 4NS, UK; \\ judit@perala.es (J.G.-G.); brunoequadros@hotmail.com (B.d.Q.); w.havelange@qmul.ac.uk (W.H.) \\ 2 Leica Biosystems Newcastle Ltd, Newcastle NE12 8EW, UK; alistair.brock@leicabiosystems.com \\ * Correspondence: c.h.brennan@qmul.ac.uk
}

Citation: García-González, J.; de Quadros, B.; Havelange, W.; Brock, A.J.; Brennan, C.H. Behavioral Effects of Developmental Exposure to JWH-018 in Wild-Type and Disrupted in Schizophrenia 1 (disc1) Mutant Zebrafish. Biomolecules 2021, 11, 319. https://doi.org/10.3390/biom11020319

Academic Editor: Enrico Moro

Received: 30 January 2021

Accepted: 16 February 2021

Published: 19 February 2021

Publisher's Note: MDPI stays neutral with regard to jurisdictional claims in published maps and institutional affiliations.

Copyright: (c) 2021 by the authors. Licensee MDPI, Basel, Switzerland. This article is an open access article distributed under the terms and conditions of the Creative Commons Attribution (CC BY) license (https:// creativecommons.org/licenses/by/ $4.0 /)$.

\begin{abstract}
Synthetic cannabinoids can cause acute adverse psychological effects, but the potential impact when exposure happens before birth is unknown. Use of synthetic cannabinoids during pregnancy may affect fetal brain development, and such effects could be moderated by the genetic makeup of an individual. Disrupted in schizophrenia 1 (DISC1) is a gene with important roles in neurodevelopment that has been associated with psychiatric disorders in pedigree analyses. Using zebrafish as a model, we investigated (1) the behavioral impact of developmental exposure to 3 MM 1-pentyl-3-(1-naphthoyl)-indole (JWH-018; a common psychoactive synthetic cannabinoid) and (2) whether disc1 moderates the effects of JWH-018. As altered anxiety responses are seen in several psychiatric disorders, we focused on zebrafish anxiety-like behavior. Zebrafish embryos were exposed to JWH-018 from one to six days post-fertilization. Anxiety-like behavior was assessed using forced light/dark and acoustic startle assays in larvae and novel tank diving in adults. Compared to controls, both acutely and developmentally exposed zebrafish larvae had impaired locomotion during the forced light/dark test, but anxiety levels and response to startle stimuli were unaltered. Adult zebrafish developmentally exposed to JWH-018 spent less time on the bottom of the tank, suggesting decreased anxiety. Loss-of-function in disc1 increased anxiety-like behavior in the tank diving assay but did not alter sensitivity to JWH-018. Results suggest developmental exposure to JWH-018 has a long-term behavioral impact in zebrafish, which is not moderated by disc1.
\end{abstract}

Keywords: zebrafish; cannabinoids; disc1; JWH-018; forced light/dark assay; anxiety

\section{Introduction}

Synthetic cannabinoids commercialized as "spice", "K2", "legal weed", or "herbal incense" are man-made chemicals used as an alternative to marijuana. They gained popularity during the early 2000s and were legal in many countries [1], with consumption prevalence ranging between $0.2 \%$ and $4 \%$ in the general population [2]. Among pregnant women, cannabis use has risen in recent years [3], and a similar trend may be true for synthetic cannabinoids, but, for the latter, prevalence estimates in pregnant women are unavailable.

The endocannabinoid system plays an important role in early brain development [4]; thus, consumption of cannabinoids (both natural or synthetic) during pregnancy may affect fetal neurodevelopment, leading to long-term behavioral alterations [5]. Delta-9tetrahydrocannabinol (THC), the major psychoactive component of cannabis, can cross the placental barrier [5], bind the cannabinoid receptors located in the fetus brain, and interfere with the endocannabinoid system affecting neurogenesis and neuronal migration [5]. Stronger effects on brain development may be produced by JWH-018 (1-pentyl-3(1-naphthoyl)-indole), one of the most common psychoactives in synthetic cannabinoids. Behavioral measures in monkeys and rats suggest JWH-018 is 4 to 8 times more potent than 
THC [6,7], and adverse outcomes associated with using synthetic cannabinoids containing JWH-018 are more frequent and severe than those arising from cannabis consumption: acute intake of JWH-018 has been shown to cause anxiety, psychosis, hallucinations, and alterations in cognitive abilities [8,9]. Given these potent adverse effects in adults, it is important to understand the short- and long-lasting consequences of JWH-018 exposure during brain development. However, such consequences still remain unknown [10].

Some genes that play important roles in neurodevelopment may modulate the effects of developmental exposure to cannabinoids. Disrupted in schizophrenia 1 (DISC1) is a gene in chromosome 1q42.1 that encodes a scaffolding protein with several protein interactions in the brain. Over 100 proteins have been suggested to interact with DISC1 [11], highlighting the pivotal role of this protein during neurodevelopment [11]. Further, DISC1 was identified in a Scottish family pedigree, where a translocation between chromosome 1 and $11(\mathrm{t}(1 ; 11)(\mathrm{q} 42.1 ; \mathrm{q} 14.3))$ segregated with psychiatric disorders including schizophrenia, depression, and bipolar disorder $[12,13]$.

There is evidence suggesting that alterations due to DISC1 loss-of-function are exacerbated by exposure to cannabinoids. Disc1 mutant mice are more susceptible to deficits in fear-associated memory after exposure to THC during adolescence [14]. Perturbation of expression of Disc1 in astrocytes, but not neurons, increased the effects of adolescent THC exposure on recognition memory assessed in adult mice [15]. Altered expression of Disc1 and THC exposure caused synergistic activation of the proinflammatory nuclear factor-k-B-cyclooxygenase-2 pathway in astrocytes, leading to secretion of glutamate and dysfunction of GABAergic neurons in the hippocampus [15]. These studies suggest that Disc1 loss-of-function exacerbates the behavioral effects of THC exposure during adolescence, but no studies have yet examined the effects on earlier developmental exposures, nor the interaction of other cannabinoids (i.e., JWH-018) with Disc1.

Here we examine the impact of developmental exposure to JWH018 on anxiety-like behavior using zebrafish as a model. Zebrafish are an ideal animal model to investigate the short- and long-lasting effects of developmental exposure to drugs of abuse: embryos develop externally and are transparent; thus, exposure can be done directly through the water, and effects can be easily monitored. High fecundity of breeding adults provides sample sizes suitable for high-throughput screening experiments with multiple treatments/doses. Although zebrafish cannot develop human psychiatric disorders, they can display behaviors that resemble stress [16], anxiety [17], or drug seeking [18], known as "intermediate phenotypes" or "endophenotypes" [19]. Effects of developmental exposure on larval behavior were assessed using the forced light/dark assay and response to acoustic startle. Adult anxiety-like behavior was assessed using the novel tank diving assay.

The forced light/dark test is a well-established behavioral assay in zebrafish larvae, where changes in locomotor activity due to alternating bright light/dark depend on the integrity of brain function and the correct development of the visual and nervous system. Transitions from dark to bright light cause an initial rapid locomotor startle response, followed by an abrupt decrease in larval movement (freezing) and subsequent progressive increase in movement that can be interpreted as a measure of recovery to stress-reactivity and anxiety [20]. Transitions from light to dark again induce a startle response, followed by an increase in locomotion and gradual return to baseline. Here, the magnitude of the startle response and the increase in locomotion on transition to light has been interpreted as a measure of anxiety-like responses.

The larval acoustic startle response has been extensively characterized and involves one of two distinct motor behaviors: a short-latency C-bend of the tail, initiating within 5-15 milliseconds of the stimulus, or a slower, long-latency C-bend response, initiating within 20-80 milliseconds. These two motor behaviors use different, possibly overlapping neuronal circuitry [21], but in this study they were measured jointly since a high-speed camera was not available.

When the abrupt sound/vibration stimuli are given repeatedly, zebrafish exhibit iterative reduction in the probability of a startle response, commonly known as habituation. 
Habituation is the mechanism by which the nervous system filters irrelevant stimuli. It is evolutionarily conserved and present in a wide range of species, from invertebrates such as Aplysia and Drosophila to vertebrates such as rodents [22]. Defective habituation is also associated with neuropsychiatric disorders, such as schizophrenia [23]. In zebrafish assays of response to acoustic startle, a progressive reduction in mean distance travelled with stimulus number gives an indication of habituation [24,25].

Novel tank diving exploits the natural tendency of zebrafish to initially stay at the bottom of a novel tank and gradually move to upper parts of the tank. The degree of "bottom dwelling" has been interpreted as an index of anxiety (greater bottom dwelling meaning greater anxiety), and it is conceptually similar to the rodent open-field and elevated plus maze tasks [17]. Other measures, such as the distance travelled in the tank during the course of the assay and the transitions to bottom of the tank, can give further insights on the hyperresponsiveness to novel environments.

Our two main aims were to interrogate whether the developing central nervous system is susceptible to the effects of JWH-018, and to investigate whether loss-of-function mutations in the disc1 gene exacerbates the effects of early developmental exposure to JWH-018. We addressed the following research questions: (1) does exposure to JWH-018 during development modulate behavior in larvae zebrafish, (2) are the effects of exposure to JWH-018 during development similar to the effects of THC or nicotine in wild-type larval zebrafish, and (3) are the acute and long-lasting effects of developmental exposure to JWH-018 or THC exacerbated by disc1 loss of function?

\section{Materials and Methods}

\subsection{Experimental Design and Timeline}

Wild-type zebrafish were exposed to $3 \mu \mathrm{M}$ JWH-018 (Tocris, Bristol, UK, Cat. No. 1342) from $24 \mathrm{~h}$ to 6 days post fertilization (dpf). At $5 \mathrm{dpf}$ (with larvae being exposed to the drug for $96 \mathrm{~h}$ ), distances travelled during forced light/dark transitions were examined. Importantly, drug was refreshed 3-5 h before behavioral testing and larvae were in the drug solution throughout the testing period. At $6 \mathrm{dpf}$ (with larvae being exposed to the drug for $120 \mathrm{~h}$ ), response and habituation to acoustic startle stimuli were examined. Larvae were also in the drug solution during the response and habituation to startle stimuli test, but in this case the drug solution was not refreshed prior to testing since the drug had been refreshed on day five.

To investigate whether the effect of JWH-018 was similar to effects of other psychoactive substances with similar usage profiles and well-characterized effects in zebrafish (namely THC and nicotine), we repeated the experimental protocol and behavioral analysis in wild-type zebrafish larvae using $2 \mu \mathrm{M}$ THC (Merck, Kenilworth, NJ, USA, Cat. No. T4764) and $0.15 \mu \mathrm{M}$ nicotine (Sigma, St. Louis, MI, USA, Cat. No. N1019). Drugs were refreshed with the same time course.

To examine the potential interactions between JWH-018 and THC exposure and disc1 mutations in the short and long term, we repeated the developmental exposure to $3 \mu \mathrm{M}$ JWH-018 and $2 \mu \mathrm{M}$ THC using disc1 wild-type and mutant zebrafish, and their behavior was assessed at 5 and $6 \mathrm{dpf}$ (as in experiments with wild-type zebrafish). Furthermore, disc1 wild-type and mutant zebrafish treated with the cannabinoids but not used for larval behavioral testing were reared to adulthood in normal conditions. At 4 months old, the anxiety-like response of the JWH-018- and THC-exposed vs non-exposed fish was assessed using the novel tank diving procedure. An overview of the study design and experimental timeline is represented in Figure 1. 


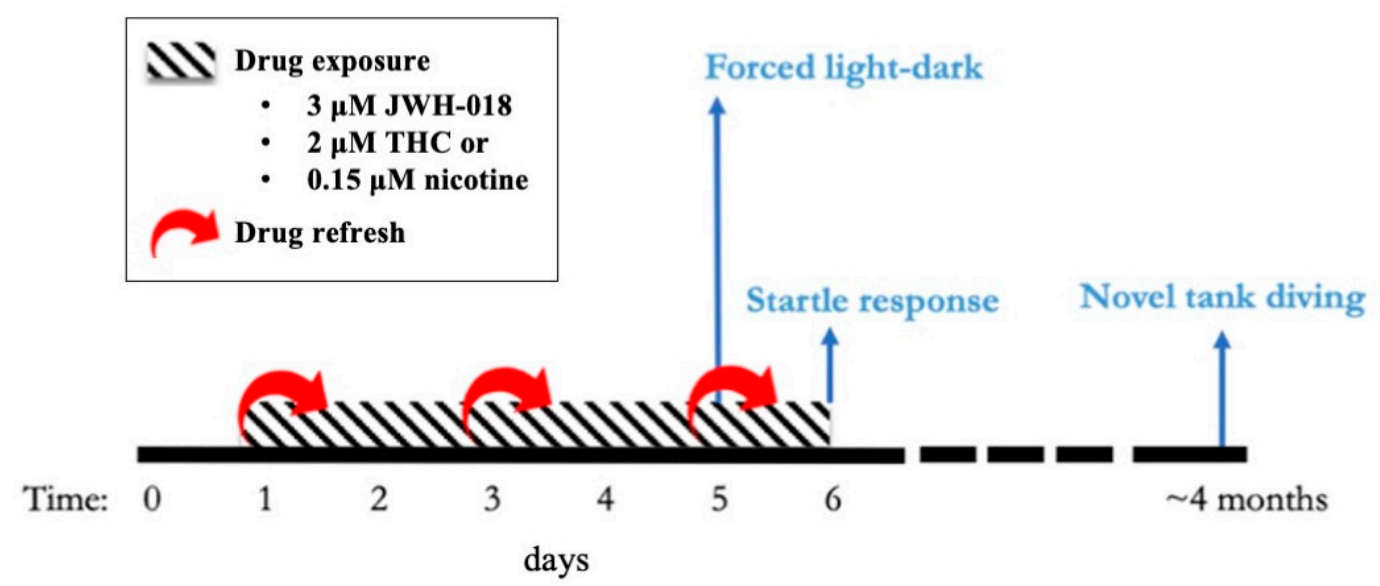

Figure 1. Experimental timeline for developmental exposure to 1-pentyl-3-(1-naphthoyl)indole (JWH-018), delta-9tetrahydrocannabinol (THC), and nicotine. The behavioral tests performed are represented in light blue.

\subsection{Animal Maintenance}

Zebrafish were housed in a recirculating system (Techniplast, UK) on a $14 \mathrm{~h}: 10 \mathrm{~h}$ light/dark cycle (08:30-22:30). The housing and testing rooms were at $\sim 25-28{ }^{\circ} \mathrm{C}$. Zebrafish were maintained in aquarium-treated water and fed three times daily with live artemia (twice) and flake food (once). Wild-type zebrafish belonged to the Tübingen strain. The disc1 line ( $\mathrm{AB}$ background strain) was obtained from the Cecilia Moens lab (Fred Hutchinson Cancer Research Center, Seattle, WA, USA) and was provided by Dr. Jon Wood (University of Sheffield). The mutant allele (disc $f^{f h 291}$ ) is caused by a point mutation in exon $2(\mathrm{~T}>\mathrm{A})$ that produces an early stop codon. More information is detailed elsewhere [26].

To breed zebrafish, we placed them in breeding tanks that had either perforated floors or a container with marbles to isolate eggs from progenitors. We moved the animals to breeding tanks in the evening and collected eggs the following morning. Eggs were incubated in Petri dishes at $28^{\circ} \mathrm{C}$ with no more than 50 embryos per dish until $5 \mathrm{dpf}$. Eggs were screened daily to ensure the absence of morphological abnormalities and consistent developmental stage across groups. If reared, larvae were moved to the recirculating system at $6 \mathrm{dpf}$ and fed with commercial fry food. The wild-type and mutant zebrafish larvae raised to adulthood were not the same as the ones used for larval behavioral analysis. The zebrafish raised to adulthood were generated by crossing wild-type and homozygous mutant adults. This enabled us to know the embryos' genotypes, and to house them separately, removing potential competitive disadvantage of mutants compared to (probably stronger) wild-type zebrafish. More than one tank for each disc1 genotype group was used to account for possible biases due to tank effects.

All procedures were carried out under license in accordance with the Animals (Scientific Procedures) Act, 1986, and under guidance from the local animal welfare and ethical review board at Queen Mary University of London.

\subsection{Developmental Drug Exposure}

2.3.1. Developmental Exposure to JWH-018, THC, and Nicotine in Wild-type Tübingen Larvae

Since JWH-018 and THC are not soluble in water, JWH-018 was dissolved in DMSO (Sigma-Aldrich, Cat. No. D8418), and THC was provided by the manufacturer in methanol $(\mathrm{MeOH})$. Care was taken to ensure that the final carrier concentration for all samples was $0.1 \%$ DMSO (for JWH-018 experiments) and $0.1 \% \mathrm{MeOH}$ (for THC experiments). To account for potential effects of the carrier substance, we used $0.1 \%$ DMSO and $0.1 \% \mathrm{MeOH}$, respectively, as control groups. Information about the half-life of THC and JWH-018 in aquatic solutions is scarce; thus, drug and control solutions were changed every $48 \mathrm{~h}$ in an attempt to ensure constant drug uptake by the zebrafish embryos and account for 
potential degradation/oxidation in the water. Zebrafish embryos usually hatch at $48 \mathrm{~h}$ post fertilization (hpf); thus, during the first hours of exposure (24 to $48 \mathrm{hpf}$ ), drugs had to penetrate the chorion. Previous research suggests that, apart from polymers with very high molecular weight [27], the chorion does not impede the uptake of chemical substances similar to JWH-018. Furthermore, the use of solvents such as DMSO has been reported to facilitate the uptake of chemicals [28].

Drug concentrations for JWH-018 and THC were chosen based on previous studies, where exposure to $2 \mu \mathrm{M}$ THC led to impaired locomotor response in zebrafish larvae [29], and $3 \mu \mathrm{M}$ JWH-018 led to behavioral alterations in rodents [30,31] and on pilot doseresponse experiments examining effects of acute exposure to JWH-018 or THC on forced light/dark responses at $5 \mathrm{dpf}$ (Supplementary Materials and Figures S3 and S4). Developmental exposure to $0.15 \mu \mathrm{M}$ nicotine was chosen because previous studies in our lab showed this dose induced increased nicotine preference in adult zebrafish (Supplementary Materials and Figure S5).

\subsubsection{Developmental Exposure to JWH-018 in disc1 Mutant Larvae}

Exposure to $3 \mu \mathrm{M}$ JWH- 018 and behavioral testing at 5 and $6 \mathrm{dpf}$ using disc1 wild-type and mutant zebrafish was carried out as for the wild-type larvae. Larvae were obtained from an in-cross of disc1 heterozygous zebrafish. Therefore, larvae were a mix of wild-type, homozygous, and heterozygous zebrafish that were randomly allocated in the experimental plates and genotyped after behavioral testing. We performed five independent experiments on five different days. To account for variation across experiments/days, the date of testing was included as a covariate in the analyses.

\subsection{Behavioral Assays}

\subsubsection{Forced Light/Dark Test}

We conducted forced light/dark tests between 9 a.m. and 4 p.m. with the drug present in the water. We placed larvae in 48 -well plates. To reduce stress due to manipulation, we let them acclimatize for at least $1 \mathrm{~h}$ in ambient light before testing. Larvae were exposed to alternating light/dark cycles of $10 \mathrm{~min}$ : there was an initial $10 \mathrm{~min}$ period of dark (baseline), followed by two cycles of $10 \mathrm{~min}$ of light and $10 \mathrm{~min}$ of dark. This protocol has been used elsewhere [32]. Distances travelled were recorded using Ethovision XT software (Noldus Information Technology, Wageningen, The Netherlands), and data were outputted in one-minute and one-second time bins. Data were fitted to linear mixed models, with total distance travelled as the response variable, experimental variables (e.g., genotype, dose, time) as fixed effects, and fish ID as random effects. Details of the data analysis are given in Supplementary Materials.

\subsubsection{Response and Habituation to Startle Stimuli Test}

We assessed the response and habituation to startle stimuli between 9 a.m. and 4 p.m. with the drug present in the water (but without drug refresh prior to the test). We used the DanioVision Observation Chamber (Noldus Information Technology, Wageningen, The Netherlands), which contains a dedicated tapping device and set the DanioVision tap stimulus at the highest intensity (intensity level: 8). Larvae were subjected to 10 sound/vibration stimuli over $10 \mathrm{~s}$ ( $1 \mathrm{~s}$ interval between each stimulus). For all experiments, distance travelled was recorded using Ethovision XT software (Noldus Information Technology, Wageningen, The Netherlands), and data were outputted in one-second time bins.

As proof of concept, we replicated the experiment by Best and colleagues [24], where 50 stimuli were given using 1, 5, and $20 \mathrm{~s}$ inter-stimulus intervals (ISI). Following the habituation paradigm [22], shorter ISI led to faster habituation (Effect of ISI: $\chi 2(2)=19.04$, $p<0.0001$ ) (Figure S1). 


\subsubsection{Novel Tank Diving Test}

We transported adult zebrafish (3-4 months) to the behavioral room in their housing tanks and let them acclimatize to the room conditions for at least $1 \mathrm{~h}$ before testing. Novel tank diving was assessed as previously described [33]: zebrafish were individually introduced into a 1.5 L trapezoid tank $(15.2 \mathrm{~cm} \times 27.9 \mathrm{~cm} \times 22.5 \mathrm{~cm} \times 7.1 \mathrm{~cm})$ (Figure S2) and filmed for $5 \mathrm{~min}$. Their behavior was tracked using EthoVision system (Noldus, Netherlands), and data were outputted in one-minute time bins. Care was taken to ensure that experimental groups were randomized during testing. Behavioral testing was conducted between 9 a.m. and 2 p.m.

We analyzed three behaviors in response to the novel tank: (1) time that zebrafish spent in the bottom third of the tank, (2) total distance that zebrafish travelled in the tank over the $5 \mathrm{~min}$, and (3) number of transitions to the top-bottom area of the tank. Details on the data analysis are in Supplementary Materials.

\subsubsection{Code Availability}

Code used to analyze the behavioral assays is available at https://github.com/ juditperala/Zebrafish-behaviour (accessed on 15 February 2021).

\subsection{Competitive Allele-Specific PCR (KASP) disc1 Larvae Genotyping}

After behavioral testing, DNA was extracted using the hot shock DNA extraction protocol. Since the loss-of-function in disc1 is caused by a point mutation (see Table 1), we used the competitive allele-specific PCR (KASP) assay (LGC, Biosearch Technologies, Hoddesdon, UK) to genotype the zebrafish.

Table 1. Genomic sequence surrounding the point loss-of-function mutation ( $\mathrm{T}>\mathrm{A}$, in red) for disc1.

\begin{tabular}{cc}
\hline Position & Genomic Sequence Surrounding the SNP Polymorphism \\
\hline & AGAGGGTTTCGAGAGAGACAACTCATCAAAGTC \\
13:49125537- 49125647 & TTCAAATAAACACCATT[T/A]GCATGATGAGGAG \\
& GACAATTTACCAGTGCAATCACGTGATGTTTTCAATT \\
\hline
\end{tabular}

\section{Results}

No obvious morphological abnormalities or impact on swimming behavior were observed after exposure to THC, JWH-018, or nicotine and prior to behavioral analysis.

\subsection{Larval Behavior during Developmental Exposure to JWH-018 in Wild-Type Zebrafish}

Over the course of the forced light/dark test, time $(\chi 2(1)=41.27, p<0.0001)$ and JWH-018 treatment $(\chi 2(1)=17.53, p<0.0001)$ predicted distance travelled by $5 \mathrm{dpf}$ larvae (Figure 2A). Exposure to $3 \mu \mathrm{M}$ JWH-018 impaired locomotion during baseline and dark periods. During the first $10 \mathrm{~min}$ of the experiment, treated larvae travelled shorter distances (mean $(\mathrm{M})=0.40, \mathrm{SE}=0.04)$ than controls $(\mathrm{M}=0.50, \mathrm{SE}=0.40)$ (Effect of treatment during baseline: $\chi 2(1)=0.04, p=0.04)$. On transition from light to dark, control larvae sharply increased their locomotion and progressively reduced it, whereas larvae treated with $3 \mu \mathrm{M}$ JWH-018 did not show as great an increase in movement $(\mathrm{M}=0.32, \mathrm{SE}=0.03)$ as controls $(\mathrm{M}=0.55, \mathrm{SE}=0.03)$ (Effect of treatment during Dark1 and Dark2: $\chi 2(1)=30.88, p<0.0001)$. On transition from dark to light period, zebrafish larvae increased the distance travelled during the first seconds after the change in light; however, no differences were observed between JWH-018 treated and non-treated larvae (Figure 2B,C). 
A)
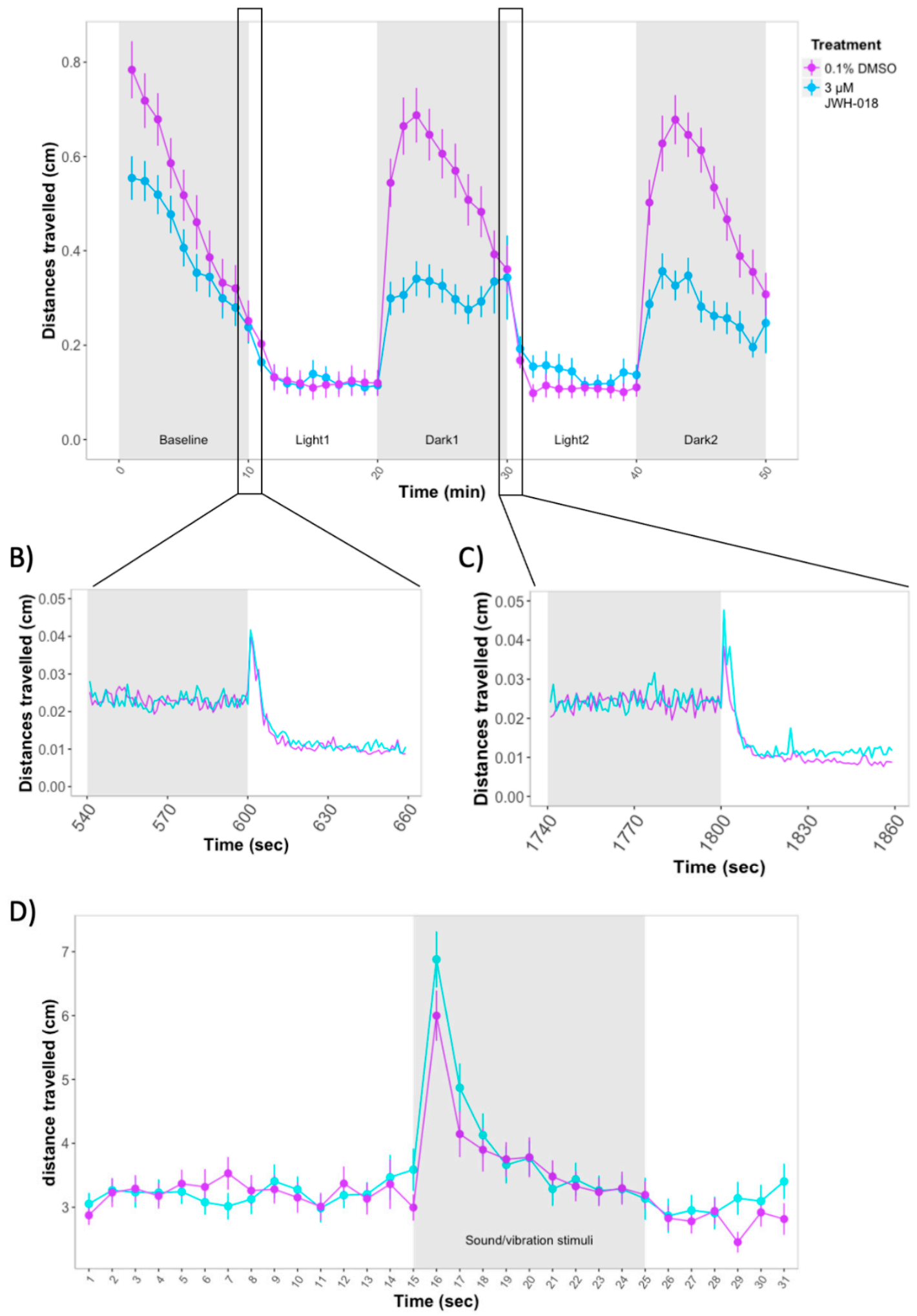

Figure 2. (A) Forced light/dark test in 5 days post fertilization (dpf) zebrafish larvae. Sample size: $n=64$ for each dose group. Each dot represents mean distance travelled per minute. Error bars represent \pm SEM. (B,C) One-second resolution plots of the transitions from dark to light during the forced light/dark test. (D) Response and habituation to acoustic startle in 6 dpf zebrafish larvae. Sample sizes: control: $n=87$, JWH-018-treated: $n=81$. Each dot represents mean distance travelled per second. Error bars represent \pm SEM. 
We assessed the rate of increase in locomotion during the light periods (measured as the slopes from minute 10 to 20 for the first light period and minute 30 to 40 for the second light period), as well as the decrease in locomotion during the dark periods (measured as the slopes from minute 23 to 30 for the first dark period and minute 43 to 50 for the second dark period). For the two light periods, there were no significant differences between the slopes of treated vs control larvae $(p>0.05)$. However, for the dark periods, the slopes were significantly steeper in control vs treated larvae (Dark1: $F(1)=7.959, p=0.006$. Dark2: $F(1)=7.966, p=0.006)$.

We next assessed the response to repeated startle stimuli at $6 \mathrm{dpf}$. There were no significant differences between $3 \mu \mathrm{M}$ JWH-018-treated and control larvae in distance travelled before and during the stimuli $(p>0.05)$ (Figure 2D).

\subsection{Larval Behavior during Developmental Exposure to THC and Nicotine in Wild-Type Zebrafish}

We investigated whether the behavioral effects of exposure to nicotine or THC during development were similar to those of JWH-018. Exposure to $2 \mu \mathrm{M}$ THC led to impaired locomotion of larvae, similar to the effects observed for the JWH-018 treatment. Distances travelled over the course of the experiment were much shorter for THC-treated larvae $(\mathrm{M}=0.62, \mathrm{SE}=0.02)$ compared to controls $(\mathrm{M}=0.91, \mathrm{SE}=0.02)($ Effect of THC treatment: $\chi 2(1)=120.89, p<0.0001)$. The differences between treated vs control larvae were consistent for baseline, light, and dark periods (Figure 3A). During the first seconds of the transitions from dark to light, zebrafish increased the distances travelled, but the increase was significantly lower in THC-treated zebrafish compared to non-treated animals (Figure 3B,C).

Treatment with $2 \mu \mathrm{M}$ THC also affected larval recovery slopes during the first light period. Slopes for control larvae were steeper $(\mathrm{M}=0.02, \mathrm{SE}=0.006)$ than for THC-treated larvae $(\mathrm{M}=0.004, \mathrm{SE}=0.006)(F(1)=5.397, p=0.0223)$. However, there were no significant differences between slopes of treated vs control larvae for the second light period or for the two dark periods $(p>0.05)$.

Zebrafish larvae treated with $2 \mu \mathrm{M}$ THC were less active during the first $30 \mathrm{~s}$ of the repeated startle response assay, before any stimulus was given (Effect of THC treatment: $\chi 2(1)=15.31, p<0.0001)$. However, during the ten sound/vibration stimuli, larvae had similar locomotor activity ( $p>0.05)$ (Figure 3D).

In contrast to JWH-018 and THC, exposure to $0.15 \mu \mathrm{M}$ nicotine produced an increase in distances travelled over the course of the forced light/dark assay $(\chi 2(1)=16.04, p<$ $0.0001)$. The increased distances travelled by nicotine-treated larvae were significant for baseline, dark, and light periods $(p<0.0001)$ (Figure $4 \mathrm{~A})$. 
A)

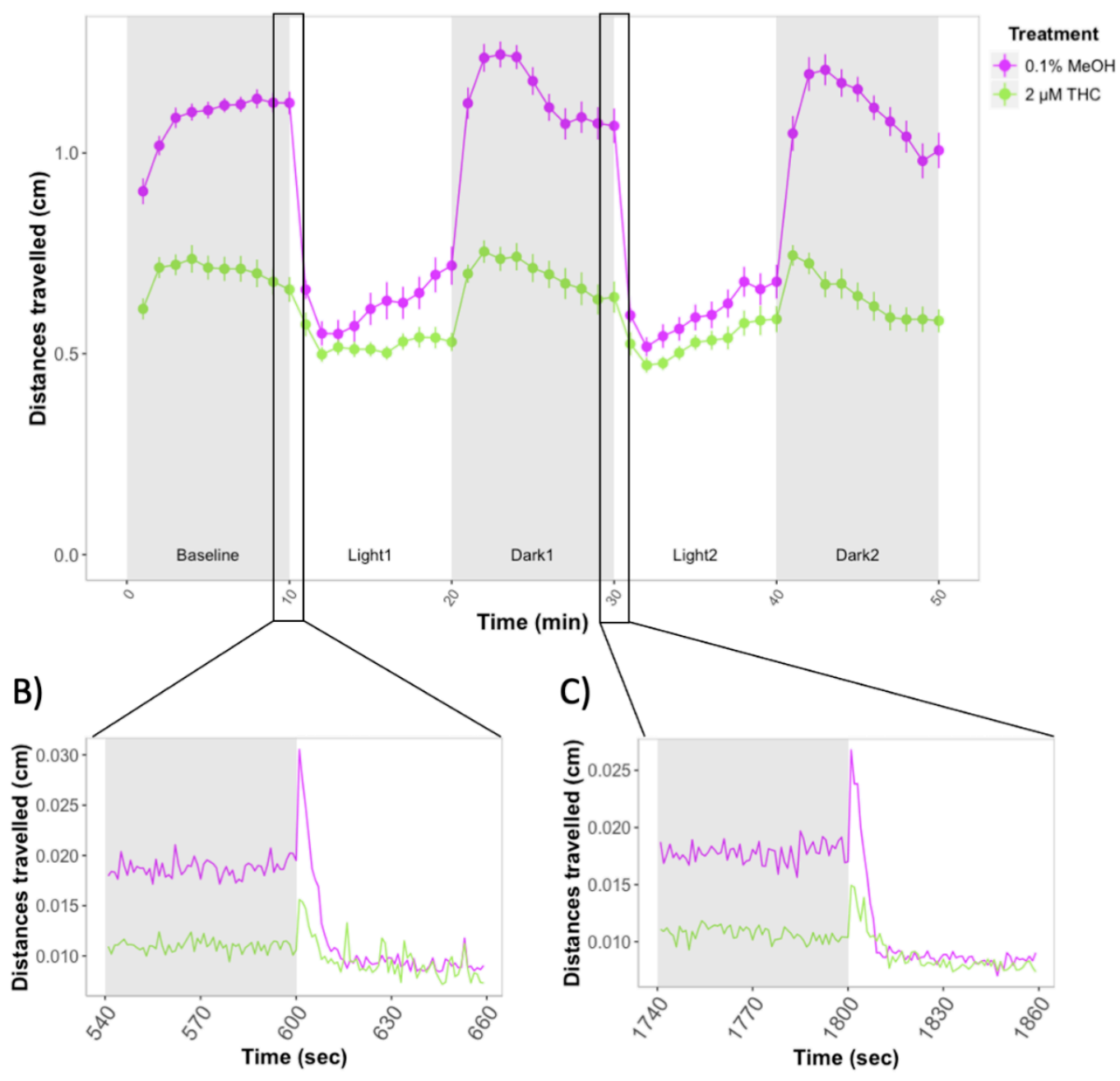

D)

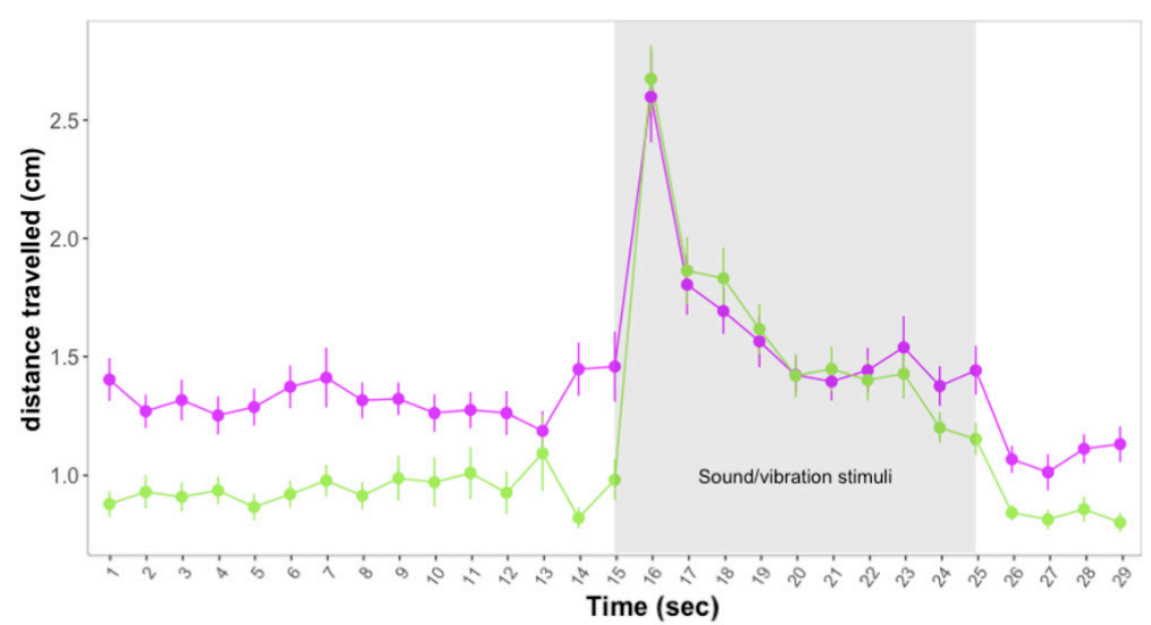

Figure 3. (A) Forced light/dark test in wild-type zebrafish exposed to $2 \mu \mathrm{M}$ THC. Sample size: $n=48$ for each dose group. Each dot represents the mean of the total distance travelled per minute. Error bars represent \pm SEM. (B,C) One-second resolution plots of the transitions from dark to light during the forced light/dark test. (D) Distances travelled by control and THC-treated larvae before and after exposure to 10 sound/vibration stimuli. Figure shows mean distances travelled in one-second time bins. Each dot represents mean distance travelled per second.Error bars represent \pm SEM. Sample sizes: $n=48$ per dose group. 

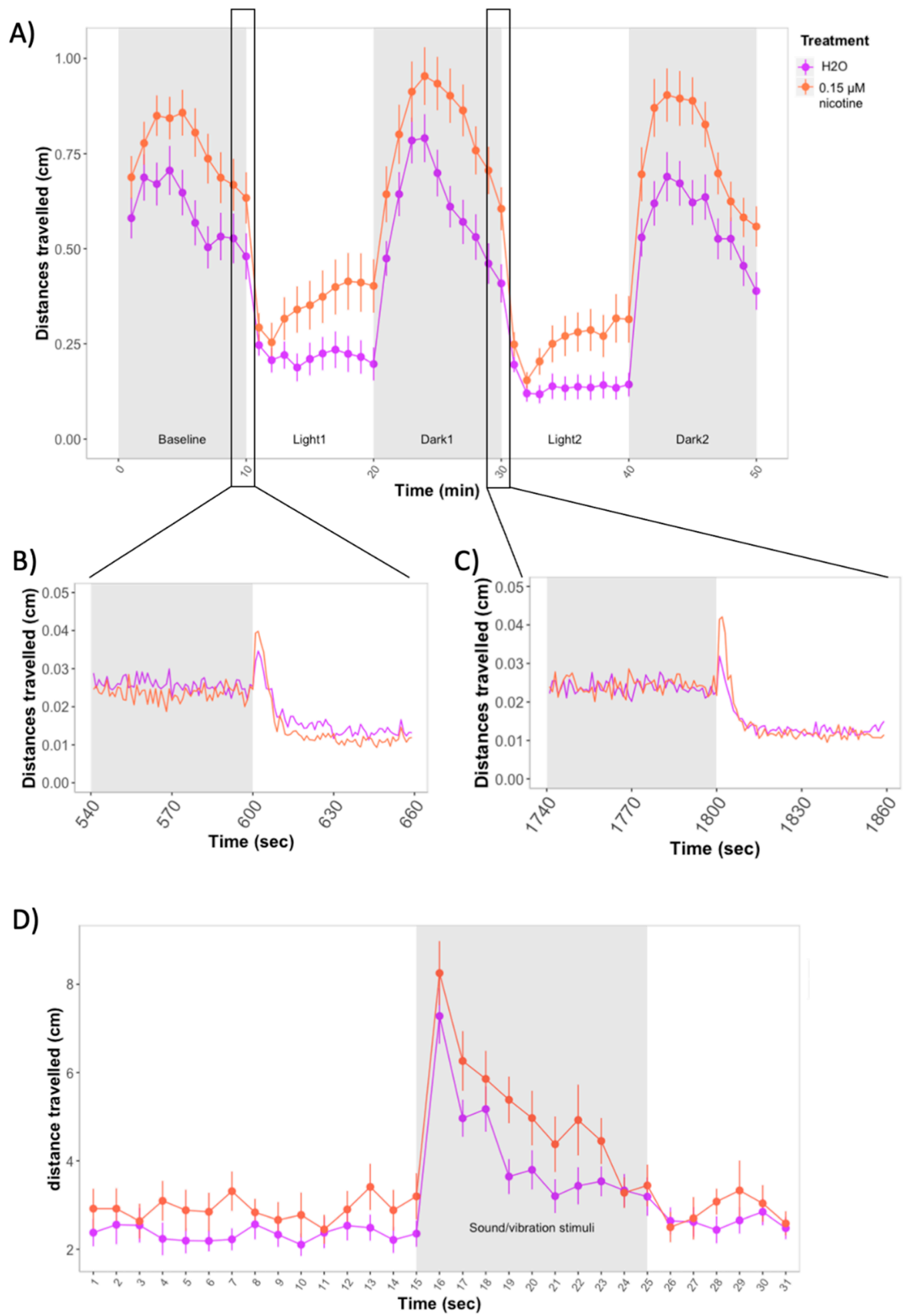

Figure 4. (A) Forced light/dark test in wild-type zebrafish exposed to $0.15 \mu \mathrm{M}$ nicotine. Sample size: $n=48$ for each dose group. Each dot represents the mean of the total distance travelled per minute. Error bars represent \pm SEM. (B,C) One-second resolution plots of the transitions from dark to light during the forced light/dark test. (D) Distances travelled by control and nicotine-treated larvae before and after exposure to 10 sound/vibration stimuli. Figure shows mean distances travelled in one-second time bins. Each dot represents mean distance travelled per second. Error bars represent \pm SEM. Control: $n=23$, treated with $0.15 \mu \mathrm{M}$ nicotine: $n=23$. 
There was a qualitative difference between control and treated zebrafish in the slopes during light periods, as nicotine-treated zebrafish seemed to recover more quickly. However, the difference in slope between nicotine-treated and control zebrafish was not significant during light nor dark periods $(p>0.05)$. During the first seconds of the transitions from dark to light, zebrafish increased the distances travelled; the increase was higher among larvae treated with nicotine compared to non-treated larvae, but differences did not reach significance (Figure $4 \mathrm{~B}, \mathrm{C}$ ).

Similar to the response seen during the forced light/dark test, zebrafish treated with $0.15 \mu \mathrm{M}$ nicotine showed increased locomotion during the acoustic startle assay. The effect was significant during stimuli $(\chi 2(1)=4.00, p=0.04)$, but not during the first 15 s before the stimuli $(p>0.05)$ (Figure 4D).

\subsection{Larval Behavior during Developmental Exposure to JWH-018 in Wild-Type and Mutant disc1 Zebrafish}

Over the $50 \mathrm{~min}$ of the forced light/dark test, JWH-018 treatment $(\chi 2(1)=12.51$, $p<0.0001)$ and time $(\chi 2(1)=72.83, p<0.0001)$ were significant predictors of distance travelled. Although disc1 wild-type larvae travelled greater distances than mutants, genotype effects were not significant $(\chi 2(1)=4.9, p=0.08)$ (Figure 5A).

During baseline, neither treatment nor genotype affected distances travelled $(p>0.05)$. During the dark periods, wild-type and disc1 homozygous (but not disc1 heterozygous larvae) travelled shorter distances when exposed to JWH-018 (Effect of JWH-018 treatment: $\chi 2(1)=16.17, p<0.0001)$. The effect of JWH-018 was also observed during the transitions from dark to light periods, where treated wild-type and disc1 mutant larvae travelled shorter distances compared to controls. However, there was no difference in the increase above baseline across treatment or genotypes (Figure 5B,C). Mutations in disc1 affected the slope during the first dark period (Effect of genotype: $\chi 2(2)=4.22, p<0.0156$ ) but not during the second one.

During light periods, there was a main effect of JWH-018 treatment $(\chi 2(1)=4.57$, $p=0.032$ ): larvae exposed to JWH-018 travelled shorter distances than control larvae. However, there were no significant main effects of disc1 genotype nor significant interactions between genotype and JWH-018 on distances travelled nor on the slopes calculated during light periods.

After $24 \mathrm{~h}$ from the last JWH-018 drug refresh, treated and control larvae showed no significant differences in distances travelled before or during the startle stimuli. There were no significant differences across disc1 genotype groups (Figure 5D).

\subsection{Larval Behavior during Developmental Exposure to THC in Wild-Type and Mutant disc1 Zebrafish}

Over the $50 \mathrm{~min}$ of the forced light/dark test, THC treatment $\left(\chi 2(1)=50.75, p=1.05 \times 10^{-12}\right)$ and disc1 genotype $\left(\chi 2(1)=45.16, p=1.56 \times 10^{-10}\right)$ were significant predictors of distance travelled (Figure 6A). THC produced a strong reduction in distances travelled across the whole experiment. The effects of THC were observed both at both one-minute and one-second time bin resolution (Figure 6B,C). The effects of disc1 genotype were dependent on the THC treatment: in drug free conditions, disc1 homozygous larvae showed increased locomotion compared to wildtype and heterozygous larvae. However, in the presence of THC, wild-type and heterozygous larvae followed the usual light/dark locomotion pattern, whereas disc1 homozygous larvae showed a steady increase in locomotion.

During light periods, THC treatment and disc1 genotype did not affect the slopes. For all genotypes in the absence of THC, distances travelled in the second light period was significantly less than in the first light period (Effect of light period number: $\chi 2(1)=62.76$, $p=2.34 \times 10^{-15}$ ). The reduction in locomotion during the light period was greater in homozygous disc1 fish and was observed only for non-treated larvae (Effect of threeway interaction between light period, disc1 genotype and THC treatment: $\chi 2(2)=61,10$, $\left.p=5.40 \times 10^{-14}\right)$. We observed a significant effect of genotype during the first dark periods 
$(\chi 2(1)=3.92, p=0.02)$ and interaction of genotype and treatment during both dark periods $($ Dark period 1: $\chi 2(1)=3.51, p=0.03$; Dark period 2: $\chi 2(1)=7.22, p=0.0009)$.

\section{A)}

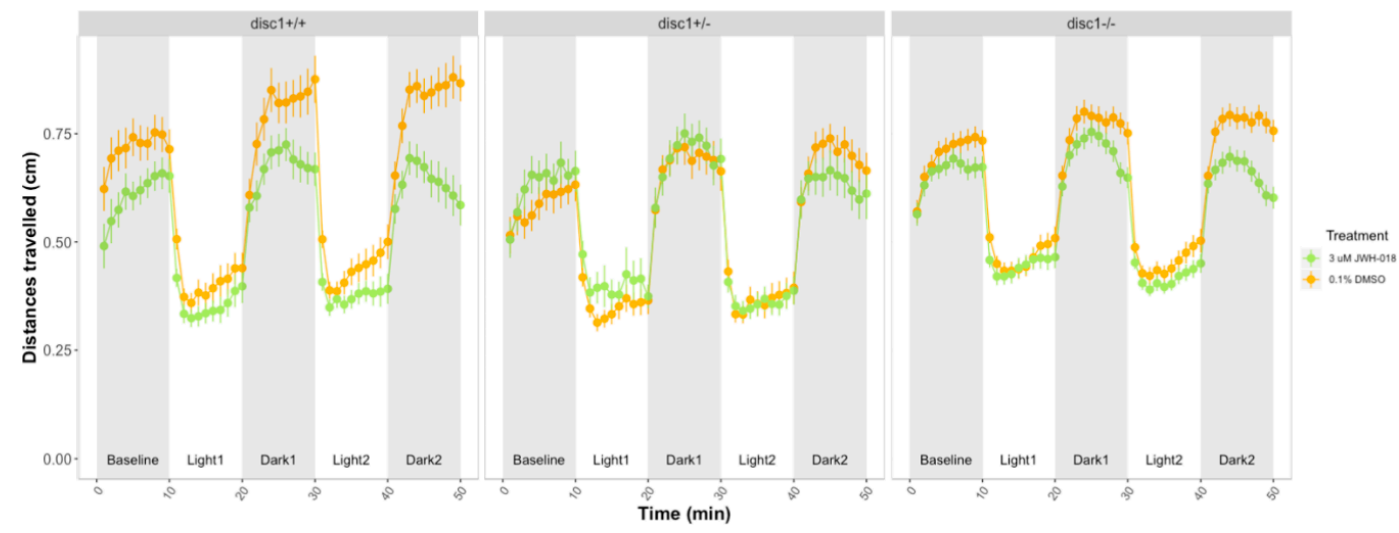

B)

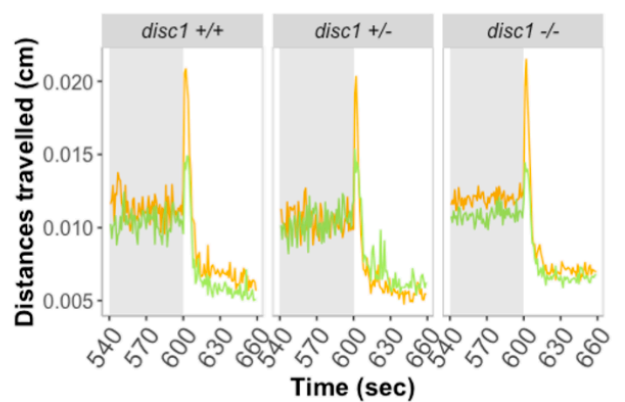

C)

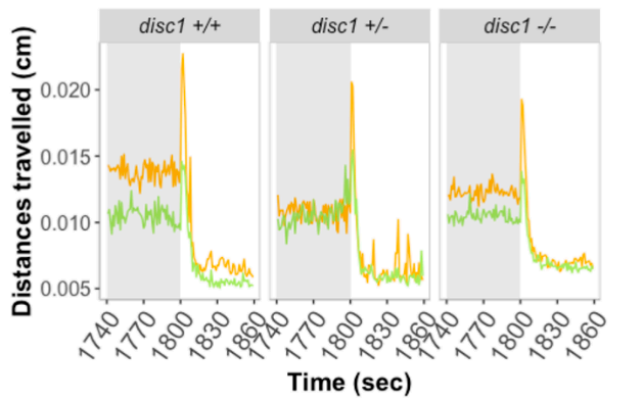

D)

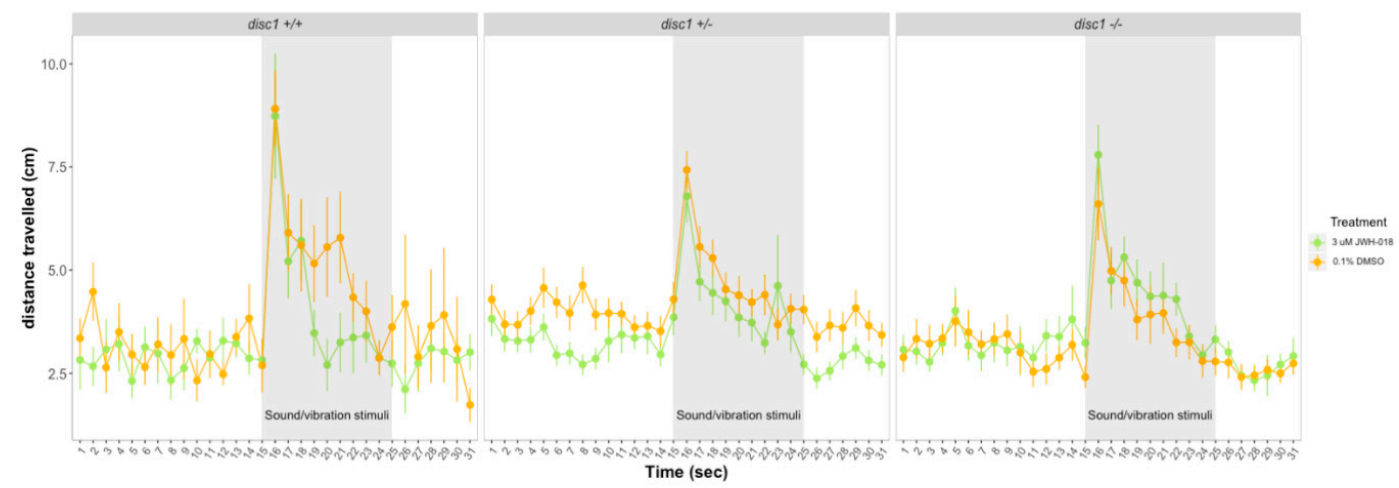

Figure 5. (A) Forced light/dark test in $5 \mathrm{dpf}$ wild-type and disc1 loss-function mutant larvae. Sample sizes for each group: control disc1 +/+: $n=30$, JWH-018 disc1 +/+: $n=34$, control disc1 +/-: $n=33$, JWH-018 disc1 +/-: $n=27$, control disc1 -/-: $n=107, \mathrm{JWH}-018$ disc1 -/-: $n=92$. Each dot represents mean distance travelled per minute. Error bars represent \pm SEM. (B,C) One-second resolution plots of the transitions from dark to light during the forced light/dark test. Each dot represents mean distance travelled per second. (D) Response and habituation to startle stimuli test in $6 \mathrm{dpf}$ control and JWH-018-treated wild-type and disc1 mutant larvae. Sample sizes: control disc1 +/ +: $n=15, \mathrm{JWH}-018$ disc1 +/ +: $n=13$, control disc1 +/-: $n=47$, JWH-018 disc1 +/-: $n=47$, control disc1 -/-: $n=22$, JWH-018 disc1 -/-: $n=22$. 


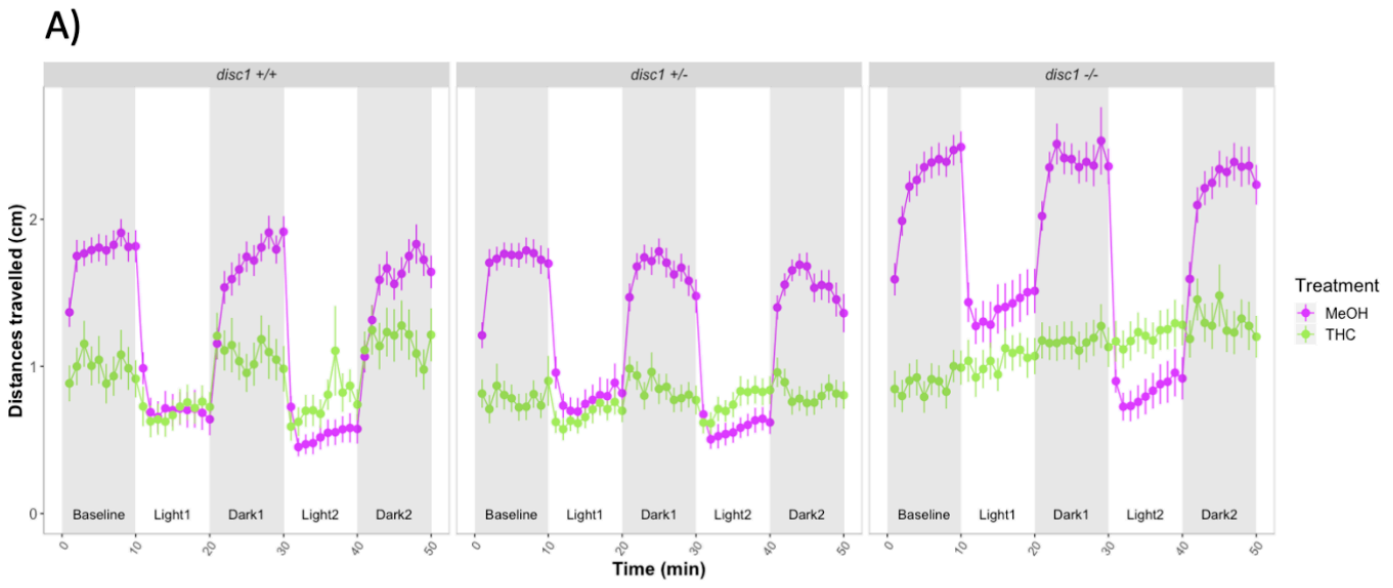

B)

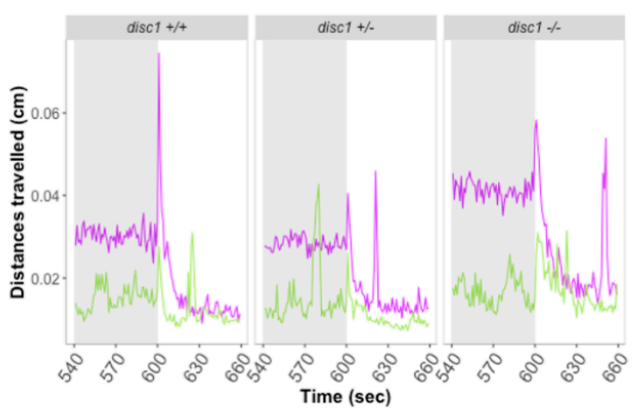

C)

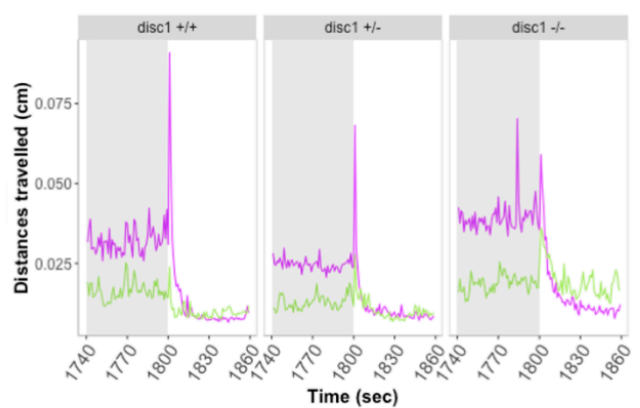

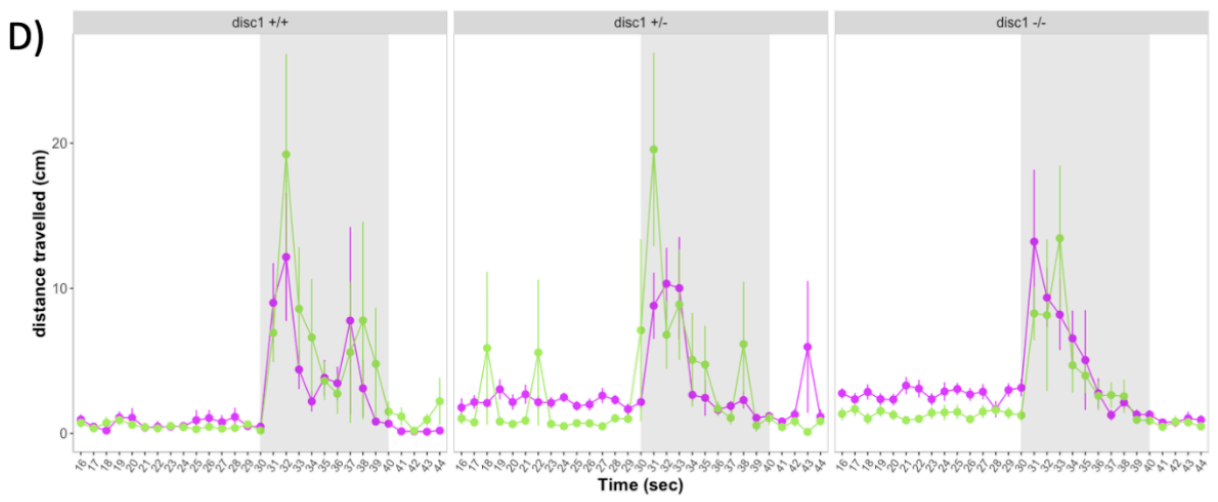

Figure 6. (A) Forced light/dark test in $5 \mathrm{dpf}$ wild-type and disc1 loss-function mutant larvae. Sample sizes for each group: control disc1 +/+: $n=39$, THC disc1 +/+: $n=29$, control disc1 +/-: $n=41$, THC disc1 +/-: $n=45$, control disc1 -/-: $n=40$, THC disc1 -/-: $n=29$. Each dot represents mean distance travelled per minute. Error bars represent \pm SEM. (B,C) One-second resolution plots of the transitions from dark to light during the forced light/dark test. Each dot represents mean distance travelled per second. (D) Response and habituation to startle stimuli test in $6 \mathrm{dpf}$ control and THC-treated wild-type and disc1 mutant larvae. Sample sizes: control disc1 +/+: $n=36$, THC disc1 +/+: $n=35$, control disc1 +/-: $n=36$, THC disc1 +/-: $n=35$, control disc1 -/-: $n=36$, THC disc1-/-: $n=36$.

There was a main effect of treatment $(\chi 2(1)=63.93, p<0.0001)$ and genotype $(\chi 2(1)=32.06$, $p<0.0001)$ on locomotion during the first $30 \mathrm{sec}$ of the repeated startle response assay, before any stimulus was given, and a Treatment $x$ Genotype interaction $(\chi 2(1)=15.67, p<0.0004)$. Wild-type larvae treated with THC did not differ in their basal movement compared to untreated larvae. However, $2 \mu \mathrm{M}$ THC exposure significantly reduced locomotion in disc1 heterozygous and homozygous mutant larvae. During the ten sound/vibration stimuli, larvae had similar locomotor activity $(p>0.05)$ (Figure 6D). 


\subsection{Adult Behavior after Developmental Exposure to JWH-018 in Wild-Type and Mutant} disc1 Zebrafish

The disc1 genotype affected the behavioral response during the novel tank assay (Figure 7). Wild-type zebrafish spent less time on the bottom of the tank than homozygous and heterozygous disc1 mutants (Effect of genotype: $\chi 2(14)=119.40, p<0.0001)$ (Figure 7A). Distances travelled over the five minutes of the experiment were also different across disc1 genotypes (Figure 7B): while wild-type zebrafish did not differ in the distance travelled over time, zebrafish heterozygous and homozygous for disc1 moved less during the first minute and increased later the distance travelled (Effect of genotype by time interaction: $\chi 2(14)=18.15, p=0.02)$. The number of transitions between the bottom and top area of the tank over the five minutes of the experiment remained similar for wild types but increased for heterozygous and homozygous zebrafish (Effect of genotype by time interaction: $\left.\chi^{2}(8)=22.93, p<0.0001\right)$ (Figure $7 C$ ).

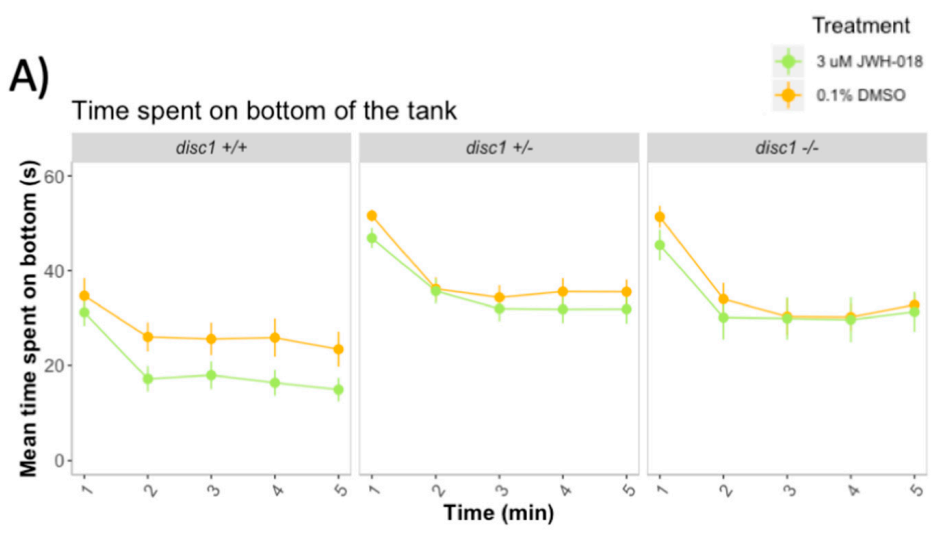

B) Cumulative distance travelled per minute

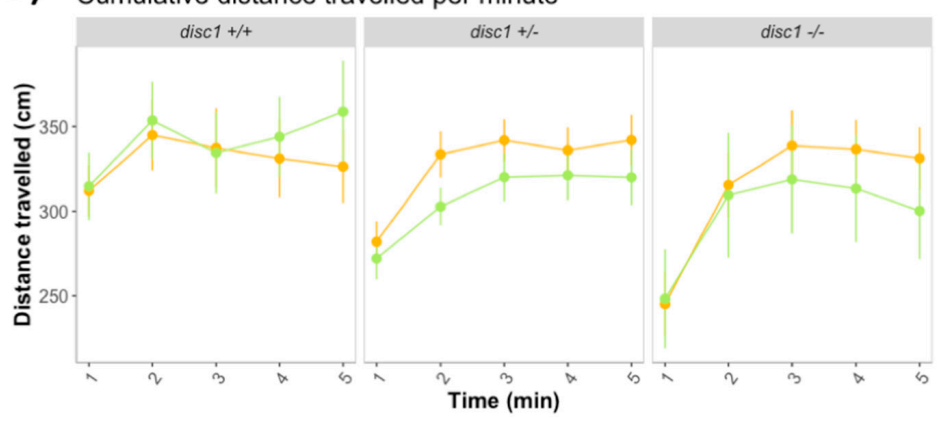

C) Number of transitions to the top-bottom area of the tank

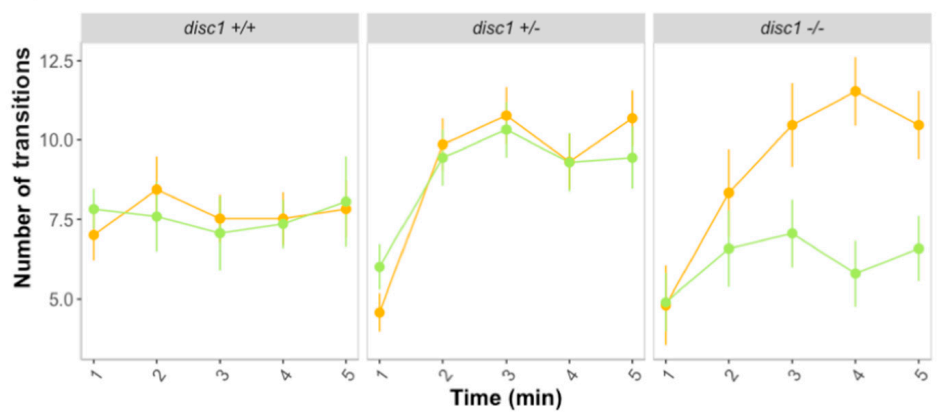

Figure 7. Novel tank diving response in adult wild-type and mutant disc1 zebrafish after developmental exposure to $3 \mu \mathrm{M}$ JWH-018. (A) Time spent on the bottom of the tank, (B) Cumulative distance travelled (C) Number of transitions between the top and bottom of the tank. Sample sizes for each group: control disc1 +/+: $n=23$, JWH-018 disc $1+/+: n=17$, control disc1 + /-: $n=35$, JWH-018 disc $1+/-: n=34$, control disc1 -/-: $n=15$, JWH-018 disc1 -/-: $n=19$. Error bars represent \pm SEM. 
Developmental exposure to JWH-018 reduced the time spent on the bottom of the tank (Effect of JWH-018 treatment: $\chi 2(1)=11.31, p<0.0001)$. The effect was stronger for wild-type than for mutant zebrafish (Figure 7A), but there were no significant genotype by JWH-018 treatment interactions $(p>0.05)$. Developmental exposure to JWH-018 did not affect the distance travelled nor the number of transitions between the top and bottom area of the tank for wild-type and heterozygous disc1 zebrafish (Figure 7B,C) $(p>0.05)$. For homozygous disc1 zebrafish, treatment with JWH-018 decreased the number of topbottom transitions but the interaction between genotype and JWH-018 treatment was not significant (Figure 7C).

In contrast to animals exposed to $3 \mu \mathrm{M}$ JWH-018, which spent less time on the bottom of the tank, developmental exposure to $2 \mu \mathrm{M}$ THC and $0.15 \mu \mathrm{M}$ nicotine had no significant effect on tank diving response in wild-type fish (Figure S6).

\section{Discussion}

This study used zebrafish as an animal model to investigate the behavioral effects of developmental exposure to JWH-018, the main psychoactive compound of synthetic cannabinoids, on larval and adult behavior in wild-type and disc1 mutant fish. Wild-type larvae exposed to JWH-18 or the related cannabinoid THC showed reduced locomotion during behavioral testing but - with the possible exception of a reduced rate of recovery on light to dark transition in a forced light/dark test in the presence of JWH-018 - their anxiety-like behavior and response to repeated sound/vibration stimuli were not altered. No locomotor effects were observed in adult wild-type fish that had been developmentally exposed to either cannabinoid, but fish exposed to JWH-018 exhibited decreased anxietylike behavior in the novel tank diving assay, suggesting that developmental exposure to JWH-018 can have lasting effects on behavior. At larval stages, loss-of-function in disc1 increased sensitivity to effects of THC but not of JWH-018. During adulthood, loss-offunction in disc1 increased zebrafish anxiety-like responses but did not moderate sensitivity to the effects of JWH-018 (See Table 2 for summary).

Table 2. Summary of results. (- - ) (++) indicate a decreased or increased response in the presence of drug, respectively.

\begin{tabular}{|c|c|c|c|c|c|}
\hline Behavioral Assay & JWH-018 & THC & $\operatorname{disc1}$ & $\begin{array}{c}\text { disc } 1 \times \text { JWH-018 } \\
\text { Interaction }\end{array}$ & $\begin{array}{c}\text { disc1 } \times \text { THC } \\
\text { Interaction }\end{array}$ \\
\hline \multicolumn{6}{|l|}{$\begin{array}{c}\text { Forced Light/Dark } \\
\text { assay at } 5 \mathrm{dpf}\end{array}$} \\
\hline Basal locomotion & $(--)$ & $(--)$ & No effect & No & Yes \\
\hline $\begin{array}{l}\text { Light period } \\
\text { locomotion }\end{array}$ & No change & $(--)$ & No effect & No & Yes \\
\hline Light period slope & No change & $(-)$ & No effect & No & No \\
\hline $\begin{array}{l}\text { Dark period } \\
\text { locomotion }\end{array}$ & $(--)$ & $(--)$ & No effect & No & Yes \\
\hline Dark period slope & $(--)$ & No change & $(-)$ & No & Yes \\
\hline \multicolumn{6}{|l|}{$\begin{array}{l}\text { Acoustic startle at } \\
6 \mathrm{dpf}\end{array}$} \\
\hline Basal locomotion & No change & $(--)$ & No effect & No & Yes \\
\hline Across taps & No change & No change & No effect & No & No \\
\hline $\begin{array}{c}\text { Adult tank } \\
\text { diving-time on } \\
\text { bottom }\end{array}$ & $(--)$ & No change & $(++)$ & No & No \\
\hline
\end{tabular}

Stimulatory and depressant responses elicited by neuroactive drugs used by humans can be modeled in zebrafish larvae. For example, exposure to adrenaline-a neurostimulant-increased the locomotor activity in the forced light/dark test, whereas tricaine- 
a CNS depressant-decreased it [34]. In this study, we show developmental exposure to JWH-018 reduced the locomotor activity of $5 \mathrm{dpf}$ wild-type zebrafish during dark periods in the forced light/dark test. The effects of JWH-018 were similar to the effects of THC but opposite to the effects of nicotine. The results for THC and nicotine are in line with previous studies showing a reduction in locomotion after exposure to THC [29] and an increase in locomotion after exposure to nicotine [35]. We hypothesize that cannabinoids may produce a CNS depressant effect, whereas exposure to nicotine has a behaviorally stimulant effect in zebrafish larvae. However, we cannot rule out that these drugs affected zebrafish behavior via impairment/activation of motor neurons or toxicity effects [36].

Alterations in the typical response of larvae to transitions between light and dark periods can be used to study the anxiety-like response in zebrafish. Others have interpreted the distance travelled in the dark period immediately following light exposure as a measure of anxiety or the distance travelled during the startle response on dark to light transitionthe greater the distance moved, the more anxious [37]. Here we found that larvae treated with JWH-018 moved significantly less than controls, suggesting possible anxiolytic effects. However, since this interpretation is problematic when there are clear effects on locomotion, we examined the slopes during both light and dark periods, which represent how quickly zebrafish larvae recover from a startle stimulus (i.e., light to dark transitions, or dark to light transitions). We would argue that this latter approach, particularly when applied to the light period, provides a measure of stress and anxiety less biased by locomotor effects, such that a more rapid rate of recovery reflects reduced anxiety. Using this interpretation, our results show JWH-018-exposed larvae recovered more slowly during the first dark period, and THC-exposed larvae recovered more slowly during the first light period-suggesting an anxiogenic effect of both cannabinoids, and larvae exposed to nicotine tended to (nonsignificantly) recover more quickly in both the light and dark-suggesting an anxiolytic effect of nicotine. Previous studies of the impact of acute exposure to THC or nicotine on the novel tank diving response in adult zebrafish support our interpretation of the forced light/dark data: compared to controls, fish pre-exposed to THC spent more time on the bottom of the tank, consistent with an anxiogenic effect [38], whereas animals pre-exposed to nicotine spent less time on the bottom of the tank, consistent with an anxiolytic effect [39]. However, as both JWH-018 and THC had marked effects on locomotion during the forced light/dark assay, caution should be taken when drawing conclusions from these data.

Using distance travelled in response to acoustic startle as an indication of anxietylevel, no effect of developmental exposure to THC or JWH-018 was observed, nor was there any effect on baseline locomotion in wild-type Tübingen larvae. As acoustic startle was conducted $24 \mathrm{~h}$ after the last drug refresh, these latter data suggest any effects on locomotion or anxiety during the forced light/dark assay are due to the presence of the drug rather than adaptive changes.

When anxiety-like behavior was assessed during adulthood, we observed wild-type zebrafish developmentally exposed to JWH-018 spent less time on the bottom of the tank, suggesting they were less anxious when placed in a new environment compared to nonexposed animals. These results differ from previous reports, suggesting anxiogenic effects due to drug withdrawal in zebrafish [40,41]. However, the time between drug exposure and analysis of behavioral effects in these withdrawal studies is very different to the current study, limiting their comparability. Further, none of the previous studies exposed fish to JWH-018, nor did they expose them at early developmental stages and test months after withdrawal. In our study, exposure to JWH-018 started at $24 \mathrm{~h}$ post fertilization, a period in which the main zebrafish brain structures (i.e., forebrain, midbrain, and hindbrain) are formed, but finer structures are still to be defined [42]. It is possible that exposures at such early ages lead to changes in gene expression and neurotransmission that impact on neurodevelopment and differ from the adaptive mechanisms occurring during other developmental periods-such as adolescence. Our results showing lack of impact of developmental exposure to nicotine on anxiety-like behavior is also at odds with findings in rodents [43] and our previous findings that developmental exposure to $0.15 \mu \mathrm{M}$ 
nicotine from 1 to 7 days led to increased sensitivity to the rewarding effects of nicotine. The reason for this difference is yet to be established, but it may reflect the relatively low concentration used and limited duration of exposure.

Although no effect of developmental exposure to nicotine or THC at the concentrations used here were observed on tank diving responses in wild-type fish, despite noticeable impact on locomotor response during exposure, the direction of the nicotine effects is in line with previous research. Our results show adult zebrafish developmentally exposed to nicotine spent more time on the bottom of the tank during the first minute of the assay, consistent with previous studies showing an increase in adult fish startle response when embryos were exposed to nicotine [44].

The novel tank diving results show that adult, but not larval, zebrafish with loss-offunction mutations in disc1 showed increased anxiety-like responses compared to wild types. These results are in line with another study showing abnormal adult stress response in this mutant line [45] and support the role of disc1 in zebrafish hypothalamus-pituitaryinterrenal axis function [45]. Previous research in zebrafish have shown that alterations in $\operatorname{disc} 1$ causes alterations in the specification of oligodendrocytes and neurons [46] and in the migration and differentiation of the neural crest (the cells that form the craniofacial cartilage and connective tissue of the head) [47]. Alterations in these processes could also underlie the alterations in behavior we observed. DISC1 is a scaffolding protein that interacts with many other proteins and regulates the formation, maintenance, and correct regulation of neural networks [11]. Given the number of interacting proteins, the specific biological mechanisms by which DISC1 acts is a complex question out of the scope of this study. However, our work supports zebrafish as a model in which to investigate the role of DISC1 in stress and neurodevelopment.

We showed no evidence of disc 1 altering sensitivity to the effects of JWH-018 at larval or adult stages; the effects of JWH-018 were less appreciable in mutant zebrafish but did not reach statistical significance. However, disc1 genotype affected larval responses to forced light/dark transition in the presence of THC and baseline locomotion in the acoustic startle assay one day after withdrawal from THC. In both cases, THC was found to have greater effects in disc1 mutants than wild-type siblings. These findings are consistent with studies in mice reporting synergistic effects between THC and alterations in Disc1. The explanation for the difference in genotype effects on response to JWH-018 compared to THC exposure is not clear. However, disparities in the psychoactive compound (JWH-018 vs THC; full vs partial agonist) and kinetics may underlie these differences. Further work using different species is needed to replicate our findings. One limitation of our study that may be of relevance in this regard is that we did not explore possible sex differences. In both human and rodent studies, females have been shown to be more sensitive to the behavioral and physiological effects of cannabinoids [48]. In addition, dominant-negative disc1 mutant mice show sex-dependent differences in social behavior and expression of cannabinoid receptors [49].

It is notable that the slopes in the untreated fish in the forced light/dark experiments differed between treatments; for example, the slope in the light period was much flatter in the JWH-018 and nicotine experiments than the THC experiments. As treatments were done in parallel balanced across plates, this difference likely reflects differences in carrier (DMSO vs methanol vs water) [50]. Interestingly, the behavioral pattern of the Tübingen wild types and the disc1 wild-type larvae in the forced light/dark test also differed in that untreated Tübingen larvae tended to show a decline in locomotion during the dark periods, whereas disc1 wild-type larvae maintained high levels of locomotion or even showed an increase over the dark period. Since they belonged to different zebrafish strains (Tübingen vs AB), differences may be due to their genetic background. It is also possible that factors such as time of day or time of year contributed to observed differences between Tübingen and disc1 strains, as the experiments were conducted separately. Although care was taken to ensure that time of drug exposure prior to testing, time of behavioral testing, 
and developmental stages were similar across experiments, these experimental parameters are known to affect zebrafish behavior [51].

JWH-018 did not affect the behavioral response of zebrafish larvae at $6 \mathrm{dpf}$. To maintain a gap of $48 \mathrm{~h}$ between each refresh, we did not refresh the drug before testing at this age, and therefore the absence of behavioral phenotype could be due to (1) JWH-018 being metabolized very quickly and no accumulation in the larvae, so after $24 \mathrm{~h}$ there was no noticeable effect and/or (2) JWH-018 is not stable in water and its psychotropic properties were lost after a few hours in the water. As our data suggest acute effects of JWH 018 are lost after $24 \mathrm{~h}$ in the water, it is possible that an altered exposure paradigm would impact behavioral outcomes. However, it is difficult to predict whether a lesser or greater effect on adult behavior would be seen: some studies for other centrally active drugs suggest an intermittent exposure regime increases adaptation [52,53]. In order to disentangle these scenarios, liquid chromatography-mass spectrometry analyses could be used to measure the concentrations of the drug in the water and in zebrafish tissue. It is also possible that the repeated administration of JWH-018 produced tolerance to behavioral effects in zebrafish larvae, since it has been shown in rodents that repeated injection of similar doses of JWH-018 produced tolerance to its hypothermic and cataleptic effects [31]. Future studies where the behavioral effect of repeated vs single exposures is compared would be valuable to examine the tolerance of different drugs.

\section{Conclusions}

This is the first study looking at the behavioral effects of early developmental exposure to JWH-018 and the interaction with loss-of-function mutations in disc1. Our results suggest that exposure to drugs of abuse during early-development leads to long-term behavioral changes in zebrafish. However, further studies in human populations and other models are needed to confirm these findings. Our results align with previous research suggesting that functional abnormalities in DISC1 have a behavioral impact and report no evidence of synergistic effect between developmental exposure to JWH-018 and disc1. These results pave the way to study molecular mechanisms by which disc1 and developmental exposure to JWH-018 act and give little evidence for interaction between disc1 and developmental exposure to synthetic cannabinoids.

Supplementary Materials: The following are available online at https://www.mdpi.com/2218-2 73X/11/2/319/s1, Figure S1: Response and habituation to startle stimuli test with different interstimulus intervals (ISI) in wild-type zebrafish larvae, Figure S2: Tank used for novel tank diving assay. Figure S3. Forced Light/Dark test in $5 \mathrm{dpf}$ wild-type zebrafish larvae after acute exposure to JWH-018 (Drug exposure: $3-4 \mathrm{~h}$ prior experiment). Sample sizes $n=40$ per experimental group. Error bars represent standard error of the mean. Figure S4. Forced Light/Dark test in $5 \mathrm{dpf}$ wildtype zebrafish larvae after acute exposure to THC (Drug exposure: $3-4 \mathrm{~h}$ prior experiment). Sample sizes $n=48$ per experimental group. Error bars represent standard error of the mean. Figure S5. $5 \mu \mathrm{M}$ nicotine-induced place preference in adult zebrafish is exacerbated by developmental exposure to $0.15 \mu \mathrm{M}$ nicotine (from $2-7 \mathrm{dpf}$ ). Sample size $n=17$ to 20 fish per experimental group. Figure S6. Novel tank diving response in adult wild-type zebrafish after developmental exposure to $2 \mu \mathrm{M}$ THC and $0.15 \mu \mathrm{M}$ nicotine. Sample sizes for each group: control THC: $n=30$, developmentally exposed to THC: $n=40$, control nicotine: $n=79$, developmentally exposed to nicotine: $n=53$. Error bars represent \pm SEM.

Author Contributions: Conceptualization, C.H.B.; methodology, C.H.B., B.d.Q., A.J.B., J.G.-G., and W.H.; formal analysis, B.d.Q., A.J.B., J.G.-G., and W.H.; investigation, C.H.B., W.H., and J.G.-G.; resources, C.H.B.; writing-original draft preparation, C.H.B. and J.G.-G.; writing-review and editing, C.H.B., J.G.-G., and W.H.; visualization, J.G.-G. and W.H.; supervision, C.H.B.; project administration, C.H.B.; funding acquisition, C.H.B. All authors have read and agreed to the published version of the manuscript.

Funding: This research was funded by NC3Rs G1000053 (C.H.B.); BBSRC BB/M007863 (C.H.B.); NIH U01 DA044400-03 (C.H.B.); and QMUL (J.G.G.). C.H.B. is a member of the Royal Society Industry Fellows' College. 
Institutional Review Board Statement: The study was conducted according to the guidelines of the Declaration of Helsinki, and approved by the Animal Welfare and Ethical Review Board, Queen Mary University of London and in accordance with Home Office license number P6D11FBCD, issued June 2019.

\section{Informed Consent Statement: Not Applicable.}

Data Availability Statement: All zebrafish data generated or analyzed during this study are included in the manuscript and supporting files. Source data files for all figures can be obtained from c.h.brennan@qmul.ac.uk or judit@perala.es.

Acknowledgments: We thank Luca Galantini for the assistance in animal maintenance and Aida Kafai Golahmadi for preliminary work on the effects of JWH-018 in zebrafish.

Conflicts of Interest: The authors declare no conflict of interest.

\section{References}

1. Castaneto, M.S.; Gorelick, D.A.; Desrosiers, N.A.; Hartman, R.L.; Pirard, S.; Huestis, M.A. Synthetic Cannabinoids: Epidemiology, Pharmacodynamics, and Clinical Implications. Drug Alcohol Depend. 2014, 144, 12-41. [CrossRef] [PubMed]

2. Loeffler, G.; Delaney, E.; Hann, M. International Trends in Spice Use: Prevalence, Motivation for Use, Relationship to Other Substances, and Perception of Use and Safety for Synthetic Cannabinoids. Brain Res. Bull. 2016, 126, 8-28. [CrossRef] [PubMed]

3. Young-Wolff, K.C.; Tucker, L.-Y.; Alexeeff, S.; Armstrong, M.A.; Conway, A.; Weisner, C.; Goler, N. Trends in Self-Reported and Biochemically Tested Marijuana Use Among Pregnant Females in California From 2009-2016. JAMA 2017, 318, $2490-2491$. [CrossRef]

4. Fride, E. The Endocannabinoid-CB Receptor System: Importance for Development and in Pediatric Disease. Neuro Endocrinol. Lett. 2004, 25, 24-30.

5. Jaques, S.C.; Kingsbury, A.; Henshcke, P.; Chomchai, C.; Clews, S.; Falconer, J.; Abdel-Latif, M.E.; Feller, J.M.; Oei, J.L. Cannabis, the Pregnant Woman and Her Child: Weeding out the Myths. J. Perinatol. 2014, 34, 417-424. [CrossRef] [PubMed]

6. Järbe, T.U.C.; Deng, H.; Vadivel, S.K.; Makriyannis, A. Cannabinergic Aminoalkylindoles, Including AM678=JWH018 Found in "Spice", Examined Using Drug ( $\Delta(9)$-Tetrahydrocannabinol) Discrimination for Rats. Behav. Pharmacol. 2011, $22,498-507$. [CrossRef]

7. Ginsburg, B.C.; Schulze, D.R.; Hruba, L.; McMahon, L.R. JWH-018 and JWH-073: $\Delta$ 9-Tetrahydrocannabinol-Like Discriminative Stimulus Effects in Monkeys. J. Pharmacol. Exp. Ther. 2012, 340, 37-45. [CrossRef] [PubMed]

8. Tait, R.J.; Caldicott, D.; Mountain, D.; Hill, S.L.; Lenton, S. A Systematic Review of Adverse Events Arising from the Use of Synthetic Cannabinoids and Their Associated Treatment. Clin. Toxicol. 2016, 54, 1-13. [CrossRef] [PubMed]

9. Theunissen, E.L.; Hutten, N.R.P.W.; Mason, N.L.; Toennes, S.W.; Kuypers, K.P.C.; de Sousa Fernandes Perna, E.B.; Ramaekers, J.G. Neurocognition and Subjective Experience Following Acute Doses of the Synthetic Cannabinoid JWH-018: A Phase 1, PlaceboControlled, Pilot Study. Br. J. Pharmacol. 2018, 175, 18-28. [CrossRef]

10. Carlier, J.; Huestis, M.A.; Zaami, S.; Pichini, S.; Busardò, F.P. Monitoring the Effects of Perinatal Cannabis and Synthetic Cannabinoid Exposure. Ther. Drug Monit. 2019. Publish Ahead of Print. [CrossRef]

11. Brandon, N.J.; Sawa, A. Linking Neurodevelopmental and Synaptic Theories of Mental Illness through DISC1. Nat. Rev. Neurosci. 2011, 12, 707-722. [CrossRef] [PubMed]

12. Jacobs, P.A.; Brunton, M.; Frackiewicz, A.; Newton, M.; Cook, P.J.L.; Robson, E.B. Studies on a Family with Three Cytogenetic Markers. Ann. Hum. Genet. 1970, 33, 325-336. [CrossRef]

13. Blackwood, D.H.R.; Fordyce, A.; Walker, M.T.; St'Clair, D.M.; Porteous, D.J.; Muir, W.J. Schizophrenia and Affective DisordersCosegregation with a Translocation at Chromosome 1q42 That Directly Disrupts Brain-Expressed Genes: Clinical and P300 Findings in a Family. Am. J. Hum. Genet. 2001, 69, 428-433. [CrossRef]

14. Ballinger, M.D.; Saito, A.; Abazyan, B.; Taniguchi, Y.; Huang, C.-H.; Ito, K.; Zhu, X.; Segal, H.; Jaaro-Peled, H.; Sawa, A.; et al. Adolescent Cannabis Exposure Interacts with Mutant DISC1 to Produce Impaired Adult Emotional Memory. Neurobiol. Dis. 2015, 82, 176-184. [CrossRef]

15. Jouroukhin, Y.; Zhu, X.; Shevelkin, A.V.; Hasegawa, Y.; Abazyan, B.; Saito, A.; Pevsner, J.; Kamiya, A.; Pletnikov, M.V. Adolescent $\Delta$ 9-Tetrahydrocannabinol Exposure and Astrocyte-Specific Genetic Vulnerability Converge on Nuclear Factor-KB-Cyclooxygenase2 Signaling to Impair Memory in Adulthood. Biol. Psychiatry 2019, 85, 891-903. [CrossRef]

16. Clark, K.J.; Boczek, N.J.; Ekker, S.C. Stressing Zebrafish for Behavioral Genetics. Rev. Neurosci 2011, 22, 49-62. [CrossRef] [PubMed]

17. Egan, R.J.; Bergner, C.L.; Hart, P.C.; Cachat, J.M.; Canavello, P.R.; Elegante, M.F.; Elkhayat, S.I.; Bartels, B.K.; Tien, A.K.; Tien, D.H.; et al. Understanding Behavioral and Physiological Phenotypes of Stress and Anxiety in Zebrafish. Behav. Brain Res. 2009, 205, 38-44. [CrossRef]

18. Bossé, G.D.; Peterson, R.T. Development of an Opioid Self-Administration Assay to Study Drug Seeking in Zebrafish. Behav. Brain Res. 2017, 335, 158-166. [CrossRef]

19. Iacono, W.G. Endophenotypes in Psychiatric Disease: Prospects and Challenges. Genome Med. 2018, 10. [CrossRef] [PubMed] 
20. Irons, T.D.; MacPhail, R.C.; Hunter, D.L.; Padilla, S. Acute Neuroactive Drug Exposures Alter Locomotor Activity in Larval Zebrafish. Neurotoxicol. Teratol. 2010, 32, 84-90. [CrossRef]

21. Burgess, H.A.; Granato, M. Sensorimotor Gating in Larval Zebrafish. J. Neurosci. 2007, 27, 4984-4994. [CrossRef]

22. Rankin, C.H.; Abrams, T.; Barry, R.J.; Bhatnagar, S.; Clayton, D.; Colombo, J.; Coppola, G.; Geyer, M.A.; Glanzman, D.L.; Marsland, S.; et al. Habituation Revisited: An Updated and Revised Description of the Behavioral Characteristics of Habituation. Neurobiol. Learn. Mem. 2009, 92, 135-138. [CrossRef]

23. Quednow, B.B.; Wagner, M.; Westheide, J.; Beckmann, K.; Bliesener, N.; Maier, W.; Kühn, K.-U. Sensorimotor Gating and Habituation of the Startle Response in Schizophrenic Patients Randomly Treated with Amisulpride or Olanzapine. Biol. Psychiatry 2006, 59, 536-545. [CrossRef] [PubMed]

24. Best, J.D.; Berghmans, S.; Hunt, J.J.F.G.; Clarke, S.C.; Fleming, A.; Goldsmith, P.; Roach, A.G. Non-Associative Learning in Larval Zebrafish. Neuropsychopharmacology 2008, 33, 1206-1215. [CrossRef]

25. García-González, J.; Brock, A.J.; Parker, M.O.; Riley, R.J.; Joliffe, D.; Sudwarts, A.; Teh, M.-T.; Busch-Nentwich, E.M.; Stemple, D.L.; Martineau, A.R.; et al. Identification of Slit3 as a Locus Affecting Nicotine Preference in Zebrafish and Human Smoking Behaviour. Elife 2020, 9. [CrossRef] [PubMed]

26. De Rienzo, G.; Bishop, J.A.; Mao, Y.; Pan, L.; Ma, T.P.; Moens, C.B.; Tsai, L.-H.; Sive, H. Disc1 Regulates Both $\beta$-Catenin-Mediated and Noncanonical Wnt Signaling during Vertebrate Embryogenesis. FASEB J. 2011, 25, 4184-4197. [CrossRef] [PubMed]

27. Henn, K.; Braunbeck, T. Dechorionation as a Tool to Improve the Fish Embryo Toxicity Test (FET) with the Zebrafish (Danio Rerio). Comp. Biochem. Physiol. C 2011, 153, 91-98. [CrossRef]

28. Kais, B.; Schneider, K.E.; Keiter, S.; Henn, K.; Ackermann, C.; Braunbeck, T. DMSO Modifies the Permeability of the Zebrafish (Danio Rerio) Chorion-Implications for the Fish Embryo Test (FET). Aquatic Toxicol. 2013, 140-141, 229-238. [CrossRef]

29. Achenbach, J.C.; Hill, J.; Hui, J.P.M.; Morash, M.G.; Berrue, F.; Ellis, L.D. Analysis of the Uptake, Metabolism, and Behavioral Effects of Cannabinoids on Zebrafish Larvae. Zebrafish 2018, 15, 349-360. [CrossRef]

30. De Luca, M.A.; Bimpisidis, Z.; Melis, M.; Marti, M.; Caboni, P.; Valentini, V.; Margiani, G.; Pintori, N.; Polis, I.; Marsicano, G.; et al. Stimulation of in Vivo Dopamine Transmission and Intravenous Self-Administration in Rats and Mice by JWH-018, a Spice Cannabinoid. Neuropharmacology 2015, 99, 705-714. [CrossRef]

31. Elmore, J.S.; Baumann, M.H. Repeated Exposure to the "Spice" Cannabinoid JWH-018 Induces Tolerance and Enhances Responsiveness to 5-HT1A Receptor Stimulation in Male Rats. Front. Psychiatry 2018, 9. [CrossRef] [PubMed]

32. Glazer, L.; Hawkey, A.B.; Wells, C.N.; Drastal, M.; Odamah, K.-A.; Behl, M.; Levin, E.D. Developmental Exposure to Low Concentrations of Organophosphate Flame Retardants Causes Life-Long Behavioral Alterations in Zebrafish. Toxicol. Sci. 2018, 165, 487-498. [CrossRef]

33. Parker, M.O.; Millington, M.E.; Combe, F.J; Brennan, C.H. Housing Conditions Differentially Affect Physiological and Behavioural Stress Responses of Zebrafish, as Well as the Response to Anxiolytics. PLoS ONE 2012, 7, e34992. [CrossRef]

34. Basnet, R.M.; Zizioli, D.; Taweedet, S.; Finazzi, D.; Memo, M. Zebrafish Larvae as a Behavioral Model in Neuropharmacology. Biomedicines 2019, 7, 23. [CrossRef]

35. Petzold, A.M.; Balciunas, D.; Sivasubbu, S.; Clark, K.J.; Bedell, V.M.; Westcot, S.E.; Myers, S.R.; Moulder, G.L.; Thomas, M.J.; Ekker, S.C. Nicotine Response Genetics in the Zebrafish. Proc. Natl. Am. Sci. USA 2009. [CrossRef]

36. Ahmed, K.T.; Amin, M.R.; Shah, P.; Ali, D.W. Motor Neuron Development in Zebrafish Is Altered by Brief (5-Hr) Exposures to THC ( $\triangle 9$-Tetrahydrocannabinol) or CBD (Cannabidiol) during Gastrulation. Sci. Rep. 2018, 8, 10518. [CrossRef]

37. Lee, H.B.; Schwab, T.L.; Sigafoos, A.N.; Gauerke, J.L.; Krug, R.G.; Serres, M.R.; Jacobs, D.C.; Cotter, R.P.; Das, B.; Petersen, M.O.; et al. Novel Zebrafish Behavioral Assay to Identify Modifiers of the Rapid, Nongenomic Stress Response. Genes Brain Behav. 2019, 18. [CrossRef] [PubMed]

38. Am, S.; Av, K. The Behavioral Effects of Acute $\Delta^{9}$-Tetrahydrocannabinol and Heroin (Diacetylmorphine) Exposure in Adult Zebrafish. Brain Res. 2013, 1543, 109-119. [CrossRef]

39. Levin, E.D.; Bencan, Z.; Cerutti, D.T. Anxiolytic Effects of Nicotine in Zebrafish. Physiol. Behav. 2007, 90, 54-58. [CrossRef] [PubMed]

40. López-Patiño, M.A.; Yu, L.; Cabral, H.; Zhdanova, I.V. Anxiogenic Effects of Cocaine Withdrawal in Zebrafish. Physiol. Behav. 2008, 93, 160-171. [CrossRef]

41. Krook, J.T.; Duperreault, E.; Newton, D.; Ross, M.S.; Hamilton, T.J. Repeated Ethanol Exposure Increases Anxiety-like Behaviour in Zebrafish during Withdrawal. PeerJ 2019, 7, e6551. [CrossRef] [PubMed]

42. Vaz, R.; Hofmeister, W.; Lindstrand, A. Zebrafish Models of Neurodevelopmental Disorders: Limitations and Benefits of Current Tools and Techniques. Int. J. Mol. Sci. 2019, 20, 1296. [CrossRef]

43. Huang, L.Z.; Liu, X.; Griffith, W.H.; Winzer-Serhan, U.H. Chronic Neonatal Nicotine Increases Anxiety but Does Not Impair Cognition in Adult Rats. Behav. Neurosci 2007, 121, 1342-1352. [CrossRef] [PubMed]

44. Eddins, D.; Cerutti, D.; Williams, P.; Linney, E.; Levin, E.D. Zebrafish Provide a Sensitive Model of Persisting Neurobehavioral Effects of Developmental Chlorpyrifos Exposure: Comparison with Nicotine and Pilocarpine Effects and Relationship to Dopamine Deficits. Neurotoxicol. Teratol 2010, 32, 99-108. [CrossRef]

45. Eachus, H.; Bright, C.; Cunliffe, V.T.; Placzek, M.; Wood, J.D.; Watt, P.J. Disrupted-in-Schizophrenia-1 Is Essential for Normal Hypothalamic-Pituitary-Interrenal (HPI) Axis Function. Hum. Mol. Genet. 2017, 26, 1992-2005. [CrossRef] [PubMed] 
46. Wood, J.D.; Bonath, F.; Kumar, S.; Ross, C.A.; Cunliffe, V.T. Disrupted-in-Schizophrenia 1 and Neuregulin 1 Are Required for the Specification of Oligodendrocytes and Neurones in the Zebrafish Brain. Hum. Mol. Genet. 2009, 18, 391-404. [CrossRef]

47. Drerup, C.M.; Wiora, H.M.; Topczewski, J.; Morris, J.A. Disc1 Regulates Foxd3 and Sox10 Expression, Affecting Neural Crest Migration and Differentiation. Development 2009, 136, 2623-2632. [CrossRef]

48. Craft, R.M. Sex Differences in Behavioral Effects of Cannabinoids. Life Sci. 2005, 77, 2471-2478. [CrossRef]

49. Kaminitz, A.; Barzilay, R.; Segal, H.; Taler, M.; Offen, D.; Gil-Ad, I.; Mechoulam, R.; Weizman, A. Dominant Negative DISC1 Mutant Mice Display Specific Social Behaviour Deficits and Aberration in BDNF and Cannabinoid Receptor Expression. World J. Biol. Psychiatry 2014, 15, 76-82. [CrossRef] [PubMed]

50. Christou, M.; Kavaliauskis, A.; Ropstad, E.; Fraser, T.W.K. DMSO Effects Larval Zebrafish (Danio Rerio) Behavior, with Additive and Interaction Effects When Combined with Positive Controls. Sci. Total. Environ. 2020, 709, 134490. [CrossRef]

51. Ogungbemi, A.; Leuthold, D.; Scholz, S.; Küster, E. Hypo- or Hyperactivity of Zebrafish Embryos Provoked by Neuroactive Substances: A Review on How Experimental Parameters Impact the Predictability of Behavior Changes. Environ. Sci. Eu. 2019, 31, 88. [CrossRef]

52. Ponzoni, L.; Braida, D.; Pucci, L.; Andrea, D.; Fasoli, F.; Manfredi, I.; Papke, R.L.; Stokes, C.; Cannazza, G.; Clementi, F.; et al. The Cytisine Derivatives, CC4 and CC26, Reduce Nicotine-Induced Conditioned Place Preference in Zebrafish by Acting on Heteromeric Neuronal Nicotinic Acetylcholine Receptors. Psychopharmacology 2014, 231, 4681-4693. [CrossRef] [PubMed]

53. Kily, L.J.M.; Cowe, Y.C.M.; Hussain, O.; Patel, S.; McElwaine, S.; Cotter, F.E.; Brennan, C.H. Gene Expression Changes in a Zebrafish Model of Drug Dependency Suggest Conservation of Neuro-Adaptation Pathways. J. Exp. Biol. 2008, 211, 1623-1634. [CrossRef] [PubMed] 